\title{
Performance Evaluation of MPPM-Coded Wireless Optical MIMO System with Combined Effects over Correlated Fading Channel
}

\author{
Yue Zhang $(\mathbb{D}$, Huiqin Wang $(\mathbb{D}$, Minghua Cao $\mathbb{D}$, and Zhongxian Bao \\ School of Computer and Communication, Lanzhou University of Technology, Lanzhou 730050, China \\ Correspondence should be addressed to Huiqin Wang; 15117024169@139.com
}

Received 6 April 2020; Accepted 16 July 2020; Published 18 August 2020

Academic Editor: Rodolfo Araneo

Copyright (c) 2020 Yue Zhang et al. This is an open access article distributed under the Creative Commons Attribution License, which permits unrestricted use, distribution, and reproduction in any medium, provided the original work is properly cited.

\begin{abstract}
The performance of wireless optical MIMO system with multiple pulse position modulation (MPPM) over correlated fading channel is investigated. The combined effects of atmospheric attenuation, atmospheric turbulence, and pointing error are taken into consideration. The bit error rate (BER) and the ergodic channel capacity are analyzed by utilizing the Poisson counting model and the exponential correlation model. Moreover, their approximate expressions are derived. The simulation results demonstrate that the pointing error is the most prominent influence factor over weak correlated channel. The performance degradation caused by a high channel correlation coefficient is more than that of pointing error in strong correlated channel. Therefore, the use of pointing, acquisition, and tracking (PAT) system and reasonable arrangement of the number and spacing of antennas at the transceiver are the keys to improve system performance.
\end{abstract}

\section{Introduction}

Wireless optical communication (WOC) has the advantages of both optical fiber links and wireless communications which has attracted a lot of attention in recent years $[1,2]$. One of the major challenges in WOC is channel fading caused by atmospheric attenuation and atmospheric turbulence [3]. Another major challenge is pointing error which cannot be neglected among the fading due to dynamic wind loads, thermal expansion, and internal vibrations [4]. To eliminate the fading caused by atmospheric channel and pointing error, a large fraction of research has focused on multiple-input multiple-output (MIMO) technology to improve the system performance [5]. The bit error rate (BER) performance and ergodic channel capacity of the WOC MIMO system with atmospheric attenuation and atmospheric turbulence have been studied in references $[6,7]$. Furthermore, the influence of misalignment characterized by the pointing error on outage probability, channel capacity, and average BER has been studied in references [8-10].

However, the aforementioned investigations have neglected the spatial correlation of MIMO subchannels. In practical WOC MIMO applications, the true benefits provided by multiple lasers may be limited by spatial correlation $[11,12]$. The spatial correlation is quantified as a function of beam separation, turbulence strength, propagation distance, and receiver aperture for the multiple-input single-output (MISO) system in [13]. Moreover, the relationship of the correlation coefficient and receiving aperture size for the MIMO system has been analyzed in reference [14]. Thereafter, the BER and the outage probability of correlated gamma-gamma fading channel have been studied in reference [15]. The results indicate that the system performance is very sensitive to the presence of spatial correlation. Therefore, spatial correlation needs to be considered to analyze WOC MIMO performance.

On the contrary, background radiation is one of the major noise sources in WOC MIMO links, and the background radiation that impacts on BER and outage capacity of typical system has been considered in reference [16]. In addition, most of the researches utilize on-off keying (OOK) and pulse position modulation (PPM) as their modulation format. As is known, OOK has lower transmission efficiency and is difficult to satisfy the requirement of high-speed transmission $[17,18]$. PPM has lower bandwidth efficiency 
and higher synchronization requirement [19]. However, MPPM gets much attention due to its advantages of higher optical power utilization and background radiation tolerance than OOK and PPM in high-speed intensity modulation/direct detection (IM/DD) communications [20]. Therefore, it is necessary to quantify the performance of MPPM-coded WOC MIMO systems. The symbol error rate (SER) or the average BER performance of the MIMO system with the MPPM has been analyzed over turbulence fading channel in references [21-23]. But the spatial correlation and the background radiation are not considered in references [20-23].

Based upon the abovementioned analysis, it could be concluded that the factors that can make impacts on the WOC MIMO applications include atmospheric attenuation, atmospheric turbulence, pointing error, spatial correlation, background radiation, and modulation format. However, to the best of our knowledge, the performance of the MPPMcoded WOC MIMO system under combined effects over correlated fading channel has not been considered in previous studies. Concerning this issue, we focus on the BER performance and the ergodic channel capacity of the MPPMcoded WOC MIMO system over correlated log-normal channel when taking the background radiation and the combined effects into consideration. Also, their approximate expressions with low complexity are derived to achieve the performance bound of the WOC MIMO system in practical communication link. The rest of this paper is organized as follows: in Section 2, theoretical analysis is proposed. In Section 3, BER and ergodic channel capacity are analyzed, and their approximate expressions with combined effects over correlated fading channel are derived. Numerical simulation results are given in Section 4, where the Monte Carlo method is utilized to investigate the performance of our proposal. Finally, a conclusion is given in Section 5.

\section{Theoretical Analysis}

2.1. Correlated Channel. The channel coefficient under the combined effects of atmospheric attenuation, atmospheric turbulence, and pointing errors can be written as

$$
h=h^{1} h^{a} h^{p}=A_{0} e^{\chi-2 R^{2} /\left(\omega^{2}-\sigma L\right)},
$$

where $h^{l}=\exp (-\sigma L)$ is the laser power attenuation with transmission distance $L, \sigma$ is the attenuation coefficient, $h^{l}$ can be considered as a constant scaling factor during a long period of time [24], $h^{a}=\exp (\chi)$ is the turbulence fading coefficient, and $\chi$ is a Gaussian random variable with mean $\mu_{\chi}$ and variance $\sigma_{\chi}^{2}$. The variance $\sigma_{\chi}^{2}=-2 \mu_{\chi}$ can be rewritten as $\sigma_{\chi}^{2}=1.23 C_{n}^{2} k^{7 / 6} L^{11 / 6} \quad$ [25]. $C_{n}^{2}$ is the refractive-index structure parameter, $k=2 \pi / \lambda$ is the wave number, and $\lambda$ is the wavelength. $h^{p} \approx A_{0} \exp \left(-2 R^{2} / \omega^{2}\right)$ is the loss caused by zero-boresight misalignment [8], $A_{0}=[\operatorname{erf}(v)]^{2}$ is the fraction of the collected power at $R=0$, and $R$ is the radial displacement at the receiver plane. $\omega^{2}=\omega_{L}^{2} \sqrt{\pi} \operatorname{erf}(v) /$ $\left[2 v \exp \left(-v^{2}\right)\right], v=\sqrt{\pi} a /\left(\sqrt{2} \omega_{L}\right), \omega_{L}$ is the beam waist at distance $L$, and $a$ is the receiving aperture radius.
It should be noted that in practical WOC MIMO systems, there is a certain spatial correlation among subchannels. Therefore, the channel coefficient matrix of the correlated channel can be defined as

$$
\mathbf{G}=\mathbf{R}_{r} \mathbf{H R}_{t}=\left[g_{n m}\right]_{N M},
$$

where $\mathbf{H}=\left[\mathbf{h}_{n m}\right]_{N M}$ is the intensity fading coefficient matrix when the subchannels are independent, $\mathbf{R}_{r}$ is the $N \times N$ receiver correlation matrix, and $\mathbf{R}_{t}$ is the $M \times M$ transmitter correlation matrix. In order to make a simple description of spatial correlation, the exponential correlation model [26] can be used to define $\mathbf{R}_{r}$ and $\mathbf{R}_{t}$ as $\mathbf{R}_{r}=\left[\mathbf{r}_{r}^{|c-b|}\right]_{c, b=1, \ldots, N}$ and $\mathbf{R}_{t}=$ $\left[\mathbf{r}_{t}^{|c-b|}\right]_{c, b=1, \ldots, M}$, where $r_{\mathrm{r}}\left(0 \leq r_{r} \leq 1\right)$ and $r_{t}\left(0 \leq r_{t} \leq 1\right)$ are the correlation coefficients of receiver and transmitter, respectively.

When the transmitter and receiver are correlated $\left(r_{r} \neq 0\right.$, $r_{t} \neq 0$ ), the channel coefficient matrix can then be expressed as

$$
\begin{aligned}
\mathbf{G}= & {\left[g_{n m}\right]_{N M}=\left[\mathbf{r}_{r}^{|c-b|}\right]_{c, b=1, \ldots N} \cdot\left[A_{0} e^{\chi_{n m}-2 R_{n m}^{2} /\left(\omega^{2}-\sigma L\right)}\right]_{N M} } \\
& \cdot\left[\mathbf{r}_{t}^{|c-b|}\right]_{c, b=1, \ldots M} .
\end{aligned}
$$

2.2. MIMO Channel. A WOC MIMO system is composed of $M$ lasers diodes (LDs) and $N$ photodetectors (PDs), as shown in Figure 1.

Assume the total transmitting power is $E_{\mathrm{s}}$, then the average power of each laser is $E_{s} / M$. When using the $Q-$ ary MPPM modulation format, a symbol period is divided into $Q$ slots. Each symbol contains $\zeta$ “on" slots $(\zeta \leq Q)$ and $Q-\zeta$ “off” slots. Therefore, there are $C_{Q}^{\zeta}=\left(\begin{array}{l}Q \\ \zeta\end{array}\right)$ number of symbols available. As a result, a subset of size $\log _{2}\left(\begin{array}{l}Q \\ \zeta\end{array}\right)$ can be selected for symbol constellation. The modulated signal that transmitted on the $m-$ th $(m=1,2, \ldots, M)$ LD can be represented by $x_{m}=\left[0,0, \ldots, A_{m}, \ldots, A_{m}, \ldots, 0\right]$, where $A_{m}$ denotes light intensity in an "on" slot and 0 denotes an "off" slot. Assume the mean of counted photons in "on" slots is $\lambda_{\text {on } \mid g_{n m}}$ and in "off" slots is $\lambda_{\text {off }}$, respectively. When the Poisson statistics model and the equal gain combination (EGC) method are utilized at the receiver end, then there are

$$
\left\{\begin{array}{l}
\lambda_{\text {on } \mid g_{n m}}=\frac{\eta E_{s}}{\varepsilon f M} \sum_{n=1}^{N} \sum_{m=1}^{M} g_{n m}+\frac{\eta N E_{b}}{\varepsilon f}, \\
\lambda_{\text {off }}=\frac{\eta N E_{b}}{\varepsilon f},
\end{array}\right.
$$

where $\eta$ is the photoelectric conversion efficiency, $f$ is the center frequency of optical carriers, $\varepsilon$ is the Planck constant, $E_{b}$ is the noise power at the PD, and $g_{n m}$ is the channel coefficient from the $m$ - th LD to the $n-$ th PD.

It can be seen from equation (4) that the simplification of $\sum_{m=1}^{M} \sum_{n=1}^{N} g_{n m}$ is important for the effective calculation of photons at the receiver. When the subchannels are correlated, the assuming that variable $D$ is equal to the $\sum_{m=1}^{M} \sum_{n=1}^{N} g_{n m}$ in equation (4) and $D$ should be written as 


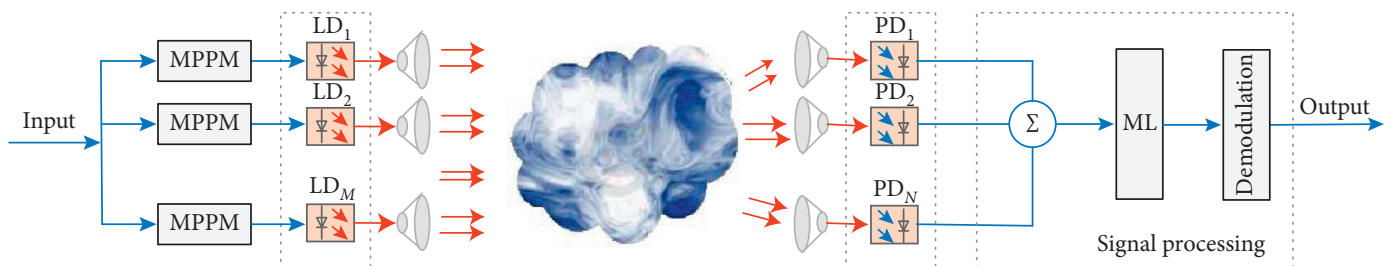

FIgUre 1: WOC MIMO system with $M$ LDs and $N$ PDs.

$$
\begin{aligned}
D & =\sum_{m=1}^{M} \sum_{n=1}^{N} g_{n m}=\sum_{m=1}^{M} \sum_{n=1}^{N}\left(r_{r}^{|c-b|}\right)_{c, b=1,2 \ldots N} \cdot\left[A_{0} \exp \left(\chi_{n m}-\frac{2 R_{n m}^{2}}{\omega^{2}-\sigma L}\right)\right] \cdot\left(r_{t}^{|c-b|}\right)_{c, b=1,2 \ldots M} \\
& =A_{0} \exp \left(-\frac{2 R^{2}}{\omega^{2}}\right) \exp (-\sigma L) \sum_{m=1}^{M} \sum_{n=1}^{N}\left(r_{r}^{|c-b|}\right)_{c, b=1,2 \ldots N} \cdot \exp \left[\chi_{n m}-\frac{2\left(R_{n m}^{2}-R^{2}\right)}{\omega^{2}}\right] \cdot\left(r_{t}^{|c-b|}\right)_{c, b=1,2 \ldots M} \\
& =A_{0} l_{0} \exp (-\delta) \sum_{m=1}^{M} \sum_{n=1}^{N}\left(r_{r}^{|c-b|}\right)_{c, b=1,2 \ldots N} \cdot \exp \left(K_{n m}\right) \cdot\left(r_{t}^{|c-b|}\right)_{c, b=1,2 \ldots M}
\end{aligned}
$$

where $l_{0}=\exp (-\sigma L), \delta=2 R^{2} / \omega^{2}$ and its probability density function (PDF) is $f_{\delta}(\delta)=\gamma^{2} e^{-\gamma^{2} \delta}, \gamma=\omega /\left(2 \sigma_{s}\right)$, and $\sigma_{s}$ is the standard deviation of the jitter offset at the receiver. The jitter variance $\sigma_{s}^{2}$ is generally used to define the intensity of pointing error. $K_{n m}=\chi_{n m}-2\left(R_{n m}^{2}-R^{2}\right) / \omega^{2}$ follows normal distribution; therefore, its mean and variance can be written as $\mu_{K_{n m}}=\mu_{\chi_{n m}}-\left(2 / \omega^{2}\right)\left\|\phi_{m}-\phi_{n}\right\|^{2}$, and $\sigma_{K_{n m}}^{2}=\sigma_{\chi_{m n}}^{2}+\left(16 \sigma_{s}^{2} / \omega^{4}\right)\left(\phi_{m}-\phi_{n}\right)^{T}\left(\phi_{m}-\phi_{n}\right)$, respectively, where $\phi_{n}\left(\phi_{n} \in \psi, \psi\right.$ is the coordinate set of all receiving apertures) denotes the central coordinate vector of the $n-$ th received laser beam.

On the contrary, when $K_{n m}$ multiplies $\mathbf{R}_{t}$ and $\mathbf{R}_{r}$, the result still follows the normal distribution. Therefore, variable $D$ can be expressed as

$$
D=A_{0} l_{0} \exp (-\delta) \sum_{k=1}^{N M} \exp \left(V_{k}\right)
$$

where $V_{k}$ follows normal distribution, and its mean and variance are $\mu_{V_{k}}=\mu_{K_{n m}} \cdot\left[\left(1-r_{\mathrm{t}}^{M}\right) /\left(1-r_{\mathrm{t}}\right)\right] \cdot\left[\left(1-r_{\mathrm{r}}^{N}\right) /\right.$ $\left.\left(1-r_{\mathrm{r}}\right)\right]$ and $\sigma_{V_{k}}^{2}=\sigma_{K_{n m}}^{2} \cdot\left[\left(1-r_{t}^{2 M}\right) /\left(1-r_{t}^{2}\right)\right] \cdot\left[\left(1-r_{r}^{2 N}\right) /\right.$ $\left.\left(1-r_{r}^{2}\right)\right]$, respectively.

The sum of log-normal variables can be approximated to another log-normal variable [27], that is, $\sum_{k=1}^{N M} \exp \left(V_{k}\right)=\exp (W)$. Therefore, variable $D$ can be simplified as

$$
D=A_{0} l_{0} \exp (W-\delta)=A_{0} l_{0} \exp (U),
$$

where $U=W-\delta, W$ is a normal distributed variable and their PDF can be written as $f_{W}(W)=\left(1 / \sqrt{2 \pi} \sigma_{W}\right) \exp$ $\left(-\left(\left(W-\mu_{W}\right)^{2} / 2 \sigma_{W}^{2}\right)\right)$. The mean $\mu_{W}=2 \ln \alpha-1 / 2 \ln \beta$ and the variance $\sigma_{W}^{2}=\ln \beta-2 \ln \alpha$, where $\alpha=\sum_{k=1}^{M N} e^{\mu_{V_{k}}+\left(\sigma_{V_{k}}^{2} / 2\right)}$, and $\beta=\sum_{k=1}^{N M} \exp \left(2 \mu_{V_{k}}+2 \sigma_{V_{k}}^{2}\right)+2 \sum_{k=1} 1^{N M-1} \sum_{j=k+1}^{N M}\{\exp$ $\left.\left(\mu_{V_{k}}+\mu_{V_{j}}\right) \exp \left[1 / 2\left(\sigma_{V_{k}}^{2}+\sigma_{V_{j}}^{2}+2 r_{k j} \sigma_{V_{k}} \sigma_{V_{j}}\right)\right]\right\}$.

As a result, $\lambda_{\text {on } \mid g_{n m}}$ can be simplified as

$$
\lambda_{\text {on } \mid D}=\frac{\eta E_{s} D}{\varepsilon f M}+\frac{\eta N E_{b}}{\varepsilon f}=\frac{\eta E_{s} A l_{0} \exp (U)}{\varepsilon f M}+\frac{\eta N E_{b}}{\varepsilon f} .
$$

The probability distribution of $U$ can be expressed as

$$
f_{U}(U)=\int_{0}^{\infty} f_{U \mid \delta}(U \mid \delta) f_{\delta}(\delta) \mathrm{d} \delta
$$

where

$$
f_{U \mid \delta}(U \mid \delta)=\frac{1}{\sqrt{2 \pi} \sigma_{W}} \exp \left\{-\frac{\left[U-\left(\mu_{W}-\delta\right)\right]^{2}}{2 \sigma_{W}^{2}}\right\} .
$$

Substituting $f_{\delta}(\delta)$ and equation (10) into equation (9), the result is

$$
f_{U}(U)=\frac{\gamma^{2}}{2} \exp \left[\frac{\gamma^{4} \sigma_{W}^{2}}{2}+\gamma^{2}\left(U-\mu_{W}\right)\right] \operatorname{erfc}\left(\frac{U+\gamma^{2} \sigma_{W}^{2}-\mu_{W}}{\sqrt{2} \sigma_{W}}\right) .
$$

Therefore, the PDF of variable $D$ can then be expressed as

$$
\begin{aligned}
f_{D}(D)= & \frac{1}{D} f_{U}(\ln D)=\frac{\gamma^{2}}{2 D} \exp \left[\frac{\gamma^{4} \sigma_{W}^{2}}{2}+\gamma^{2}\left(\ln D-\mu_{W}\right)\right] \\
& \cdot \operatorname{erfc}\left(\frac{\ln D+\gamma^{2} \sigma_{W}^{2}-\mu_{W}}{\sqrt{2} \sigma_{W}}\right)
\end{aligned}
$$

It should be noted that the cumulative distribution of intensity attenuation coefficients is no longer a log-normal distribution under the combined effects. It follows a distribution composed of coefficients, complementary error function, and exponential function.

\section{Performance Analysis}

3.1. Maximum Likelihood (ML) Detection. Assume $Z=\left\{z_{n q}, n=1,2, \ldots, N ; q=1,2, \ldots, Q\right\}$ is a set of received 
observations, where $z_{n q}$ denotes the observed photoelectrons of $n$-thPD in time slot $q$. When employing the EGC method, there is $z_{q}=\sum_{n=1}^{N} z_{n q}$. If one of the $\left(\begin{array}{c}Q \\ \zeta\end{array}\right)$ binary patterns in the $x_{k}$ is launched, then the value detected by ML can be written as

$$
\widehat{x}_{k}=\arg \max _{x_{k}} f\left(Z \mid x_{k}\right)=\arg \max _{x_{k}} \prod_{q \in Q_{\text {on }}^{(k)}} \frac{\exp \left(-\lambda_{\text {on } \mid g_{n m}}\right)\left(\lambda_{\text {on } \mid g_{n m}}\right)^{z_{q}}}{z_{q} !} \prod_{q \in Q_{o f f}^{(k)}} \frac{\exp \left(-\lambda_{\text {off }}\right)\left(\lambda_{\text {off }}\right)^{z_{q}}}{z_{q} !},
$$

where $Q_{\text {on }}^{(k)}$ and $Q_{\text {off }}^{(k)}$ denote the $k$ - th"on" and "off" slot, respectively. $z_{q}$ !, $\exp \left(-\lambda_{\text {on } \mid g_{n m}}\right)$, and $\exp \left(-\lambda_{\text {off }}\right)$ are constant to $x_{k}$. Therefore, equation (13) can be simplified by taking the logarithm as

$$
\begin{aligned}
\widehat{x}_{k} & =\arg \max _{x_{k}} \prod_{q \in Q_{o n}^{(k)}}\left(\lambda_{\text {on } \mid g_{n m}}\right)^{z_{q}} \prod_{q \in Q_{\text {off }}^{(k)}}\left(\lambda_{\text {off }}\right)^{z_{q}}=\arg \max _{x_{k}}\left[\sum_{q \in Q_{o n}^{(k)}} z_{q} \ln \left(\lambda_{\text {on }} \mid g_{n m}\right)+\sum_{q \in Q_{\text {off }}^{(k)}} z_{q} \ln \left(\lambda_{\text {off }}\right)\right] \\
& =\arg \max _{x_{k}}\left[\sum_{q \in Q_{\text {on }}^{(k)}} z_{q} \ln \left(\frac{\eta E_{s}}{\varepsilon f M} \sum_{m=1}^{M} \sum_{n=1}^{N} g_{n m}+\frac{\eta N E_{b}}{\varepsilon f}\right)+\sum_{q \in Q_{\text {off }}^{(k)}} z_{q} \ln \left(\frac{\eta N E_{b}}{\varepsilon f}\right)\right] .
\end{aligned}
$$

Since $\sum_{m=1}^{M} \sum_{n=1}^{N} g_{n m}$ is simplified to $D$, equation (14) can be written as

$$
\widehat{x}_{k}=\arg \max _{x_{k}}\left[\sum_{q \in Q_{\text {on }}^{(k)}} z_{q} \ln \left(\lambda_{\text {on } \mid D}\right)+\sum_{q \in Q_{\text {off }}^{(k)}} z_{q} \ln \left(\lambda_{\text {off }}\right)\right]=\arg \max _{x_{k}}\left[\sum_{q \in Q_{\text {on }}^{(k)}} z_{q} \ln \left(\frac{\eta E_{s} D}{\varepsilon f M}+\frac{\eta N E_{b}}{\varepsilon f}\right)+\sum_{q \in Q_{\text {off }}^{(k)}} z_{q} \ln \left(\frac{\eta N E_{b}}{\varepsilon f}\right)\right] .
$$

The results and discussion may be presented separately, or in one combined section, and may optionally be divided into headed subsections.

3.2. Bit Error Rate Performance. Assume the signal passes through a slow fading channel, which means the channel fading coefficient is constant in a symbol period. Taking the background radiation into consideration, the possible errors fall into two categories: the definite error and the indefinite error.
3.2.1. Definite Error Probability. The definite error probability can be defined as that the number of photoelectrons detected in an "off" slot is higher than that in an "on" slot. Let $\left\{S_{\text {on }, l}, \quad l=1, \ldots, \zeta\right\}$ and $\left\{S_{\text {off }, l}, \quad l=1, \ldots, Q-\zeta\right\}$ denote the sum of photoelectrons count in "on" and "off" slots, respectively. The definite error probability can be expressed as

$$
\begin{aligned}
P_{\mathrm{def} \mid g_{n m}} & =\sum_{u=0}^{\infty} P\left[\left(\left|\left\{l: S_{\text {on } \mid g_{n m}, l}=u\right\}\right|=i\right) \cap\left(\left|\left\{l: S_{\text {on } \mid g_{n m}, l}>u\right\}\right|=\zeta-i\right) \cap\left(\left|\left\{l: S_{\text {off }, l}>u\right\}\right|=j\right) \cap\left(\left|\left\{l: S_{\text {off }, l} \leq u\right\}\right|=Q-\zeta-j\right)\right] \\
& =\sum_{u=0}^{\infty} \sum_{i=1}^{\zeta}\left(\begin{array}{l}
\zeta \\
i
\end{array}\right) P\left(S_{\text {on } \mid g_{n m}}=u\right)^{i} P\left(S_{\text {on } \mid g_{n m}}>u\right)^{\zeta-i} \times \sum_{j=1}^{Q-\zeta}\left(\begin{array}{c}
Q-\zeta \\
j
\end{array}\right) P\left(S_{\text {off }}>u\right)^{j} P\left(S_{\text {off }} \leq u\right)^{Q-\zeta-j} .
\end{aligned}
$$


Since the received photoelectrons in "on" and "off" slots follow Poisson distribution, which can be described as

$$
\begin{cases}P\left(S_{\text {on } \mid g_{n m}}=\mu\right)=\frac{\lambda_{\text {on } \mid g_{n m}}^{\mu} e^{-\lambda_{\text {on } \mid g_{n m}}}}{\mu !}, & \mu=0,1,2, \ldots, \\ P\left(S_{\text {on } \mid g_{n m}} \leq \mu\right)=\sum_{b=0}^{\mu} \frac{\lambda_{\text {on } \mid g_{n m}}^{b} e^{-\lambda_{\text {on } \mid g_{n m}}}}{b !}, & \mu=0,1,2, \ldots\end{cases}
$$

$$
P_{\operatorname{def} \mid g_{n m}}=\sum_{u=0}^{\infty} \sum_{i=1}^{\zeta}\left(\begin{array}{l}
\zeta \\
i
\end{array}\right)\left(\frac{\lambda_{\text {on } \mid g_{n m}} e^{-\lambda_{\text {on } \mid g_{n m}}}}{\mu !}\right)^{i}\left(1-\sum_{b=0}^{\mu} \frac{\lambda_{\text {on } \mid g_{n m}}^{b} e^{-\lambda_{\text {on } \mid g_{n m}}}}{b !}\right)^{\zeta-i} \cdot \sum_{j=1}^{\mathrm{Q}-\zeta}\left(\begin{array}{c}
Q-\zeta \\
j
\end{array}\right)\left(1-\sum_{b=0}^{\mu} \frac{\lambda_{\text {off }}^{b} e^{-\lambda_{\text {off }}}}{b !}\right)^{j}\left(\sum_{b=0}^{\mu} \frac{\lambda_{\text {off }}^{b} e^{-\lambda_{\text {off }}}}{b !}\right)^{Q-\zeta-j} .
$$

3.2.2. Indefinite Error Probability. The indefinite error probability means that one or more of the "off" slots has the same photoelectrons count as the lowest-valued "on" slots.
Thus, equation (16) can be rewritten as
As a result, the receiver is likely to make a wrong decision. The indefinite error probability can be expressed as

$$
\begin{aligned}
& P_{\text {indef } \mid g_{n m}}=\sum_{u=0}^{\infty} \sum_{i=1}^{\zeta}\left(\begin{array}{c}
\zeta \\
i
\end{array}\right) P\left(S_{\text {on } \mid g_{n m}}=\mu\right)^{i} P\left(S_{\text {on } \mid g_{n m}}>\mu\right)^{\zeta-i} \cdot \sum_{t=1}^{\mathrm{Q}-\zeta} \frac{\left(\begin{array}{c}
t+i \\
i
\end{array}\right)^{-1}}{\left(\begin{array}{c}
t+i \\
i
\end{array}\right)}\left(\begin{array}{c}
Q-\zeta \\
t
\end{array}\right) P\left(S_{\text {off }}=\mu\right)^{t} P\left(S_{\text {off }}<\mu\right)^{Q-\zeta-t}
\end{aligned}
$$

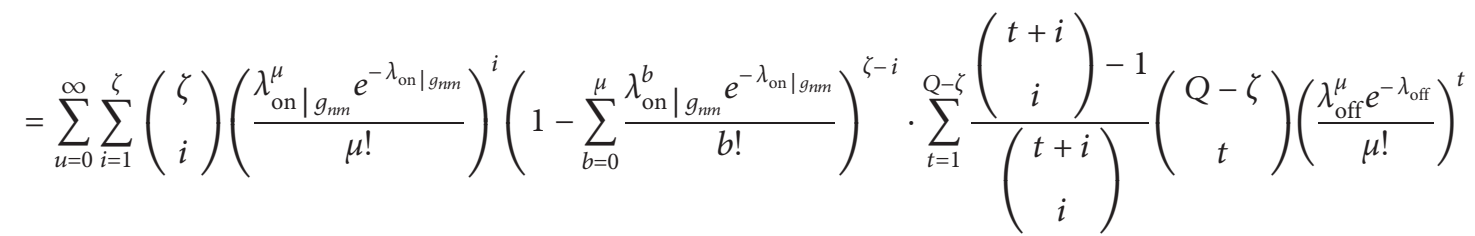

$$
\begin{aligned}
& \cdot\left(\sum_{b=0}^{\mu-1} \frac{\lambda_{\mathrm{off}}^{b} e^{-\lambda_{\mathrm{off}}}}{b !}\right)^{Q-\zeta-t}
\end{aligned}
$$

As a result, the conditional probability of symbol error with background radiation and channel fading can be written as

$$
\begin{aligned}
P_{s \mid g_{n m}}= & P_{\mathrm{def} \mid g_{n m}}+P_{\text {indef } \mid g_{n m}}=\sum_{u=0}^{\infty} \sum_{i=1}^{\zeta}\left(\begin{array}{c}
\zeta \\
i
\end{array}\right)\left(\frac{\lambda_{\text {on } \mid g_{n m}}^{\mu} e^{-\lambda_{\text {on } \mid g_{n m}}}}{\mu !}\right)^{i}\left(1-\sum_{b=0}^{\mu} \frac{\lambda_{\text {on } \mid g_{n m}}^{b} e^{-\lambda_{\text {on } \mid g_{n m}}}}{b !}\right)^{\zeta-i} \\
& \cdot\left[\sum_{j=1}^{Q-\zeta}\left(\begin{array}{c}
Q-\zeta \\
j
\end{array}\right)\left(1-\sum_{b=0}^{\mu} \frac{\lambda_{\text {off }}^{b} e^{-\lambda_{\text {off }}}}{b !}\right)^{j}\left(\sum_{b=0}^{\mu} \frac{\lambda_{\text {off }}^{b} e^{-\lambda_{\text {off }}}}{b !}\right)^{Q-\zeta-j}+\sum_{t=1}^{Q-\zeta} \frac{\left(\begin{array}{c}
t+i \\
i
\end{array}\right)-1}{\left(\begin{array}{c}
t+i \\
i
\end{array}\right)}\left(\begin{array}{c}
Q-\zeta \\
t
\end{array}\right)\left(\frac{\lambda_{\text {off }}^{\mu} e^{-\lambda_{\text {off }}}}{\mu !}\right)^{t}\left(\sum_{b=0}^{\mu-1} \frac{\lambda_{\text {off }}^{b} e^{-\lambda_{\text {off }}}}{b !}\right)^{Q-\zeta-t}\right] .
\end{aligned}
$$


Equation (20) shows the conditional probability of symbol error depends on the sum of channel fading coefficients $\sum_{m=1}^{M} \sum_{n=1}^{N} g_{n m}$. Therefore, the theoretical probability of symbol error is

$$
\begin{aligned}
& P_{s}=\underbrace{\int_{-\infty}^{+\infty} \cdots \int_{-\infty}^{+\infty}}_{M N} P_{s \mid g_{n m}} f\left(g_{n m}\right) \mathrm{d} g_{n m} \\
& =\left[\sum_{j=1}^{Q-\zeta}\left(\begin{array}{c}
Q-\zeta \\
j
\end{array}\right)\left(1-\sum_{b=0}^{\mu} \frac{\lambda_{\mathrm{off}}^{b} e^{-\lambda_{\mathrm{off} f}}}{b !}\right)^{j}\left(\sum_{b=0}^{\mu} \frac{\lambda_{\mathrm{off}}^{b} e^{-\lambda_{\mathrm{off}}}}{b !}\right)^{Q-\zeta-j}+\sum_{t=1}^{\mathrm{Q}-\zeta} \frac{\left(\begin{array}{c}
t+i \\
i
\end{array}\right)-1}{\left(\begin{array}{c}
t+i \\
i
\end{array}\right)}\left(\begin{array}{c}
Q-\zeta \\
t
\end{array}\right)\left(\frac{\lambda_{\mathrm{off}}^{\mu} e^{-\lambda_{\mathrm{off}}}}{\mu !}\right)^{t}\left(\sum_{b=0}^{\mu-1} \frac{\lambda_{\mathrm{off}}^{b} e^{-\lambda_{\mathrm{off}}}}{b !}\right)^{Q-\zeta-t}\right]
\end{aligned}
$$

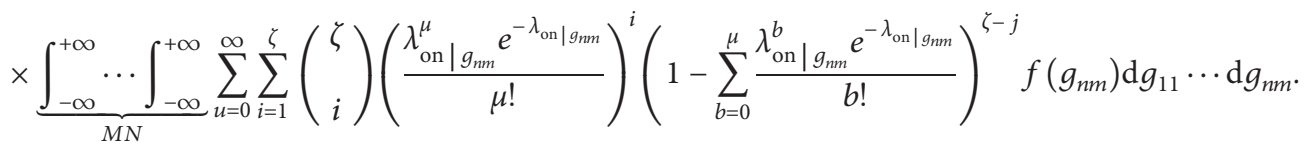

$$
P_{s}=\int_{-\infty}^{\infty} P_{s \mid D} f(D) \mathrm{d} D
$$

Because the sum of channel coefficients $g_{n m}$ can be simplified to a variable $D$, the approximate probability of symbol error with background radiation can be written as
Considering that $P_{b}=[Q / 2(Q-1)] P_{s}$, the approximate BER can be expressed as

$$
\begin{aligned}
P_{b}= & \frac{Q}{2(Q-1)}\left[\sum_{j=1}^{Q-\zeta}\left(\begin{array}{c}
Q-\zeta \\
j
\end{array}\right)\left(1-\sum_{b=0}^{\mu} \frac{\lambda_{\mathrm{off}}^{b} e^{-\lambda_{\text {off }}}}{b !}\right)^{j}\left(\sum_{b=0}^{\mu} \frac{\lambda_{\mathrm{off}}^{b} e^{-\lambda_{\text {off }}}}{b !}\right)^{Q-\zeta-j}+\sum_{t=1}^{Q-\zeta} \frac{\left(\begin{array}{c}
t+i \\
i
\end{array}\right)-1}{\left(\begin{array}{c}
t+i \\
i
\end{array}\right)}\left(\begin{array}{c}
Q-\zeta \\
t
\end{array}\right)\left(\frac{\lambda_{\mathrm{off}}^{\mu} e^{-\lambda_{\mathrm{off}}}}{\mu !}\right)^{t}\left(\sum_{b=0}^{\mu-1} \frac{\lambda_{\mathrm{off}}^{b} e^{-\lambda_{\mathrm{off}}}}{b !}\right)^{Q-\zeta-t}\right] \\
& \int_{-\infty}^{+\infty} \sum_{u=0}^{\infty} \sum_{i=1}^{\zeta}\left(\begin{array}{c}
\zeta \\
i
\end{array}\right)\left(\frac{\lambda_{\mathrm{on} \mid D}^{\mu} e^{-\lambda_{\mathrm{on} \mid D}}}{\mu !}\right)^{i}\left(1-\sum_{b=0}^{\mu} \frac{\lambda_{\mathrm{on} \mid D}^{b} e^{-\lambda_{\mathrm{on} \mid D}}}{b !}\right)^{\zeta-i} \frac{\gamma^{2}}{2 D} \exp \left(\frac{\gamma^{4} \sigma_{W}^{2}}{2}+\gamma^{2}\left(\ln D-\mu_{W}\right)\right) \operatorname{erfc}\left(\frac{\ln D+\gamma^{2} \sigma_{W}^{2}-\mu_{W}}{\sqrt{2} \sigma_{W}}\right) \mathrm{d} D .
\end{aligned}
$$

Equation (23) reveals that the approximate BER upper bound related to the parameters of $Q, \zeta, M, N, E_{s}, E_{\mathrm{b}}, \gamma$, and $D$. It should be noted that the parameters $l_{0}, r_{t}, r_{r}, \sigma_{s}^{2}$, and $\sigma_{\chi_{n m}}^{2}$ are factors which can influence $D$.
3.3. Ergodic Channel Capacity. According to equation (4), the channel capacity of the wireless optical MIMO system can be defined by

$$
C=\max I(x ; y),
$$

where $x$ and $y$ denote the launched and received signal. The instantaneous channel capacity can be written as

$$
C=\log _{2}\left(\begin{array}{c}
Q \\
\zeta
\end{array}\right)-\sum_{i=1}^{\zeta} \sum_{d=0}^{\zeta-i}(-1)^{d}\left(\begin{array}{c}
\zeta \\
i
\end{array}\right)\left(\begin{array}{c}
\zeta-i \\
d
\end{array}\right) \log _{2}\left(\begin{array}{c}
Q-\zeta+i \\
i
\end{array}\right) \exp \left(\begin{array}{c}
\eta E_{s}(i+d) \sum_{m} \sum_{n} g_{n m} \\
\varepsilon f M
\end{array}\right)
$$

Considering the channel coefficient is a random variable. Therefore, the ergodic channel capacity is usually utilized to analyze the system capacity. As a result, the theoretical
MPPM-coded ergodic channel capacity of the WOC MIMO system can be expressed as 


$$
\begin{aligned}
& C_{\text {avg }}=\log _{2}\left(\begin{array}{c}
Q \\
\zeta
\end{array}\right)-\underbrace{\int_{0}^{\infty} \cdots}_{M N} \int_{0}^{\infty} \sum_{i=1}^{\zeta} \sum_{d=0}^{\zeta-i}(-1)^{d}\left(\begin{array}{c}
\zeta \\
i
\end{array}\right)\left(\begin{array}{c}
\zeta-i \\
d
\end{array}\right) \log _{2}\left(\begin{array}{c}
Q-\zeta+i \\
i
\end{array}\right) \exp \left(\begin{array}{c}
\eta E_{s}(i+d) \sum_{m} \sum_{n} g_{n m} \\
\varepsilon f M
\end{array}\right) \\
& f\left(g_{11}\right) \cdots f\left(g_{N M}\right) \underbrace{d g_{11} \cdots d g_{N M}}_{M N} .
\end{aligned}
$$

It is complex to perform the $M \times N-$ fold integration. As mentioned in Section 2.2, the sum of $\sum_{m=1}^{M} \sum_{n=1}^{N} g_{n m}$ can be approximated as variable $D$. Thus, equation (26) can be simplified as

$$
C_{\text {avg }}=\log _{2}\left(\begin{array}{c}
Q \\
\zeta
\end{array}\right)-\sum_{i=1}^{\zeta} \sum_{d=0}^{\zeta-i}(-1)^{d}\left(\begin{array}{c}
\zeta \\
i
\end{array}\right)\left(\begin{array}{c}
\zeta-i \\
d
\end{array}\right) \log _{2}\left(\begin{array}{c}
Q-\zeta+i \\
i
\end{array}\right) \cdot \int_{0}^{\infty} \exp \left(-\frac{\eta E_{s}(i+d) D}{\varepsilon f M}\right) f(D) \mathrm{d} D
$$

The approximation is much simpler than its former version since it only requires a single integration. Substituting equation (12) into equation (27), the approximate ergodic channel capacity under combined effects can be further expressed as

$$
\begin{aligned}
C_{\text {avg }}= & \log _{2}\left(\begin{array}{c}
Q \\
\zeta
\end{array}\right)-\sum_{i=1}^{\zeta} \sum_{d=0}^{\zeta-i}(-1)^{d}\left(\begin{array}{c}
\zeta \\
i
\end{array}\right)\left(\begin{array}{c}
\zeta-i \\
d
\end{array}\right) \log _{2}\left(\begin{array}{c}
Q-\zeta+i \\
i
\end{array}\right) \cdot \int_{0}^{\infty} \frac{\gamma^{2}}{2 D} \exp \left(\frac{\gamma^{4} \sigma_{W}^{2}}{2}+\gamma^{2}\left(\ln D-\mu_{W}\right)+\frac{\eta E_{s}(i+d) D}{\varepsilon f M}\right) \\
& \operatorname{erfc}\left(\frac{\ln D+\gamma^{2} \sigma_{W}^{2}-\mu_{W}}{\sqrt{2} \sigma_{W}}\right) \mathrm{d} D .
\end{aligned}
$$

Equation (28) is a simplification of equation (26). It is evident from equation (28) that the ergodic channel capacity is related to parameters as $Q, \zeta, \varepsilon, f, E_{s}, M$, and $D$, where $l_{0}, \sigma_{\chi_{n m}}^{2}, \sigma_{s}^{2}$ and $r_{r}, r_{t}$ are factors which can influence $D$.

\section{Numerical Investigation}

In this section, the BER and the ergodic channel capacity of the WOC MIMO system with combined effects over correlated fading channel have been numerically investigated based on the foregoing analysis. Especially, the BER curves are obtained by Monte Carlo simulations, and the curves of ergodic channel capacity are based on the equations. Moreover, all the derived expressions are verified via simulations. Numerical simulations are carried out by MATLAB with parameters as $\eta=0.5, Q=5$, $\zeta=2, \lambda=1550 \mathrm{~nm}, l_{0}=0.9, \quad L=1000 \mathrm{~m}, E_{b}=-170 \mathrm{dBJ}$, $\sigma_{s}^{2}=0.1, C_{n}^{2}=2.23 \times 10^{-14}$, and $\sigma_{\chi_{n m}}^{2}=0.3$. The turbulence fading and misalignment fading are characterized by parameters $\sigma_{x}^{2}$ and $\sigma_{s}^{2}$.

Table 1 demonstrates the 5-ary MPPM symbol constellation with 2 active time slots. It is evident from the table that only 2 time slots need to be activated when sending 3 bit information during a symbol period.

Figure 2 shows the BER curves under combined effects, where CE denotes the condition of combined effects in independent identically distributed (iid) channel, cor denotes the correlated channel, appro denotes the approximate value of the proposed method, and exact denotes the theoretical value. It is observed that the approximate BER performances are slightly worse than the corresponding theoretical value. Note that the computation complexity is nearly $75 \%$ lower than that of the theoretical one. Therefore, such an approximation is appropriate for practical application.

Figure 3 shows the approximate BER performance of a $2 \times 2$ WOC MIMO system, where Per denotes the ideal condition, $\mathrm{AT}$ denotes the condition only with atmospheric turbulence, and AT\&AA denotes the condition with both the atmospheric turbulence and atmospheric attenuation. The result shows that the CE seriously impacts on the system performance. When BER $=10^{-4}$, the required energy increases by about $24 \mathrm{dBJ}$ compared with the Per case. It should be noted that the pointing error leads to the most significant impact on the BER performance among all the influencing factors. For example, when $\mathrm{BER}=10^{-4}$, the required energy for $\mathrm{CE}$ is $22.5 \mathrm{dBJ}$ more than that of AT\&AA. Therefore, the pointing error is the most prominent influence factor over independent channel.

Figure 4 demonstrates the approximate BER curves versus $E_{s}$ under different correlation coefficients in a $2 \times 2$ WOC MIMO system. It can be observed that the higher the 
TABLE 1: MPPM symbol constellation.

\begin{tabular}{|c|c|c|c|c|}
\hline Bits & 000 & 001 & 010 & 011 \\
\hline Modulation & $x_{m}=\left[0,0,0, A_{m}, A_{m}\right]$ & $x_{m}=\left[0,0, A_{m}, A_{m}, 0\right]$ & $x_{m}=\left[A_{m}, A_{m}, 0,0,0\right]$ & $x_{m}=\left[0, A_{m}, A_{m}, 0,0\right]$ \\
\hline $\begin{array}{l}\text { Bits } \\
\text { Modulation }\end{array}$ & $x_{m}=\left[0, A_{m}, 0,0, A_{m}\right]$ & $x_{m}=\left[0,0, A_{m}, 0, A_{m}\right]$ & $x_{m}=\left[A_{m}^{110}, 0, A_{m}, 0,0\right]$ & $x_{m}=\left[0, A_{m}, 0, A_{m}, 0\right]$ \\
\hline
\end{tabular}

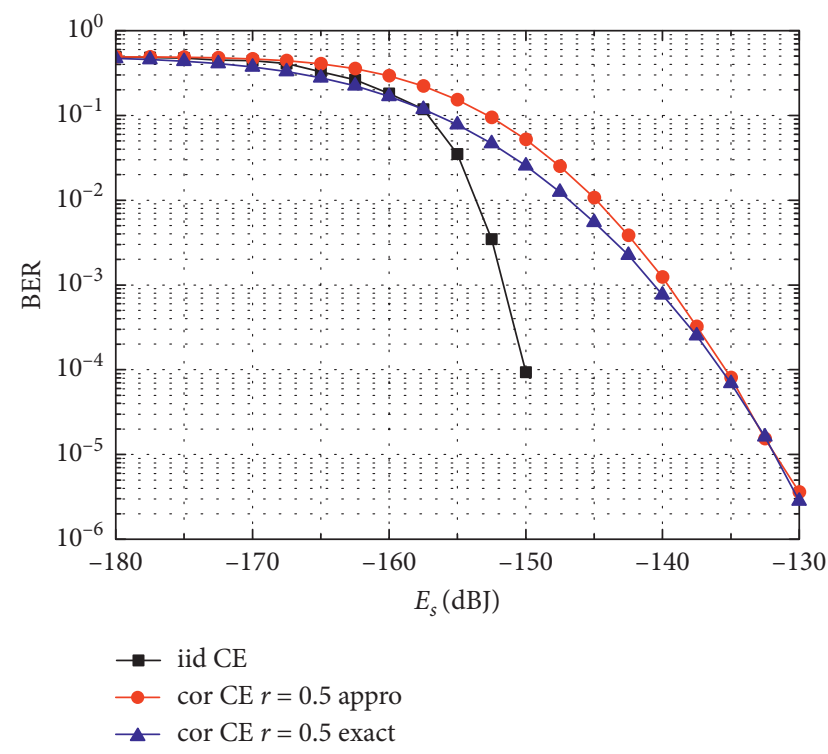

FIgURE 2: The exact and approximate of BER under combined effects.

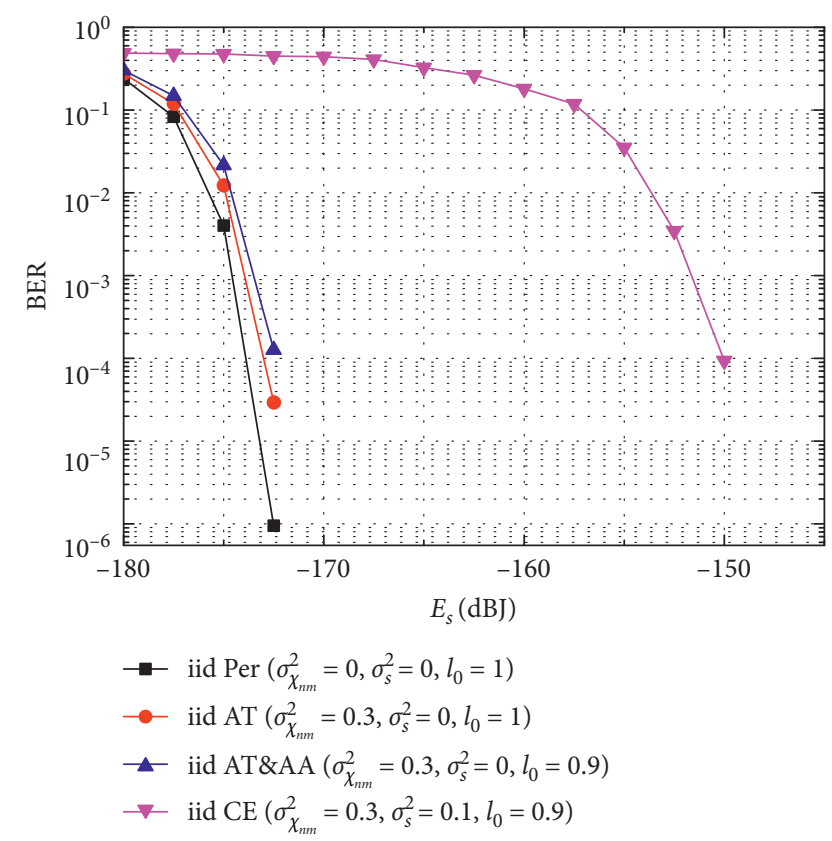

Figure 3: BER of different conditions over independent channel in a $2 \times 2$ MIMO system.

correlation coefficient, the worse the BER performance. When BER $=10^{-4}$ and the correlated coefficients are $0.3,0.5$, and 0.8 , the required energy increases by about $10 \mathrm{dBJ}$, $15 \mathrm{dBJ}$, and $28 \mathrm{dBJ}$, respectively, compared with independent channel. Particularly, the BER goes to its maximum when the correlation coefficient is 1 , which can be regarded as the upper bound of the system BER. Moreover, it is evident that the strong channel correlation can seriously impact on the system BER performance. For example, when BER $=10^{-4}$, the power loss caused by the combined effects is $24 \mathrm{dBJ}$, while the power loss caused by strong correlation $(r \geq 0.8)$ is $28 \mathrm{dBJ}$. Therefore, high channel correlation coefficientcaused performance degradation is more than that of combined effects in strong correlated channel. According to Figures 3 and 4, the BER performance of the WOC MIMO system is seriously affected by pointing error over independent and weak correlated channel, while the spatial correlation is the most prominent influence factor over strong correlated channel.

Figure 5 shows the approximate BER performance under combined effects over correlated channel with different turbulence $\left(\sigma_{\chi_{n m}}^{2}\right)$ and pointing error jitter variance $\left(\sigma_{s}^{2}\right)$. It is evident from the plot that the increased $\sigma_{\chi_{n m}}^{2}$ and $\sigma_{s}^{2}$ lead to worse BER, where $\sigma_{s}^{2}$ plays a more prominent role in the process. For example, at $\mathrm{BER}=10^{-4}$, the required energy increases by about $0.1 \mathrm{dBJ}$ and $10 \mathrm{dBJ}$, when $\sigma_{\chi_{n m}}^{2}$ and $\sigma_{s}^{2}$ increase from 0.1 to 0.3 , respectively. Therefore, the increase of $\sigma_{s}^{2}$ has a greater impact on the BER performance of the WOC MIMO system than the increase of $\sigma_{\chi_{n m}}^{2}$.

Figure 6 shows the BER performance of PPM and MPPM formats in a $2 \times 2$ MIMO system. It is evident from the plot that the BER performance of $(Q=5, \zeta=2) \mathrm{MPPM}$ is better than that of 4-PPM and 8-PPM formats over correlated channel. The reason for this is that, for activated pulse combination of MPPM, the redundancy resulting from the constellation causes stronger anti-interference ability. In addition, the spectrum efficiency of $(Q=5, \zeta=2)$ MPPM is $0.6 \mathrm{bits} / \mathrm{s} / \mathrm{Hz}$, which is $0.1 \mathrm{bits} / \mathrm{s} / \mathrm{Hz}$ and $0.225 \mathrm{bits} / \mathrm{s} / \mathrm{Hz}$ higher than that of 4-PPM and 8-PPM, respectively.

Figure 7 shows the ergodic channel capacity of PPM and MPPM formats in a $2 \times 2 \mathrm{MIMO}$ system. It is clear that the ergodic channel capacity of $(Q=5, \zeta=2)$ MPPM is 3.4 bits/ channel, which is $1.4 \mathrm{bits} / \mathrm{channel}$ and $0.4 \mathrm{bits} / \mathrm{channel}$ higher than that of 4-PPM and 8-PPM, respectively. Comparing the BER, ergodic channel capacity, and spectrum efficiency of PPM and MPPM, it can be obtained that MPPM has advantages in the wireless optical MIMO communication system.

Figure 8 shows the theoretical and approximate ergodic channel capacity with different number of "on" slots. Both theoretical (equation (26)) and approximate (equation (28)) expressions are depicted in the figure. It is observed that the performance of approximation is slightly worse than the corresponding theoretical one. Note that the computation complexity of approximation is much lower. Therefore, the slight performance loss is acceptable for the approximation. 




FIGURE 4: BER of different correlation coefficients in a $2 \times 2$ MIMO system.

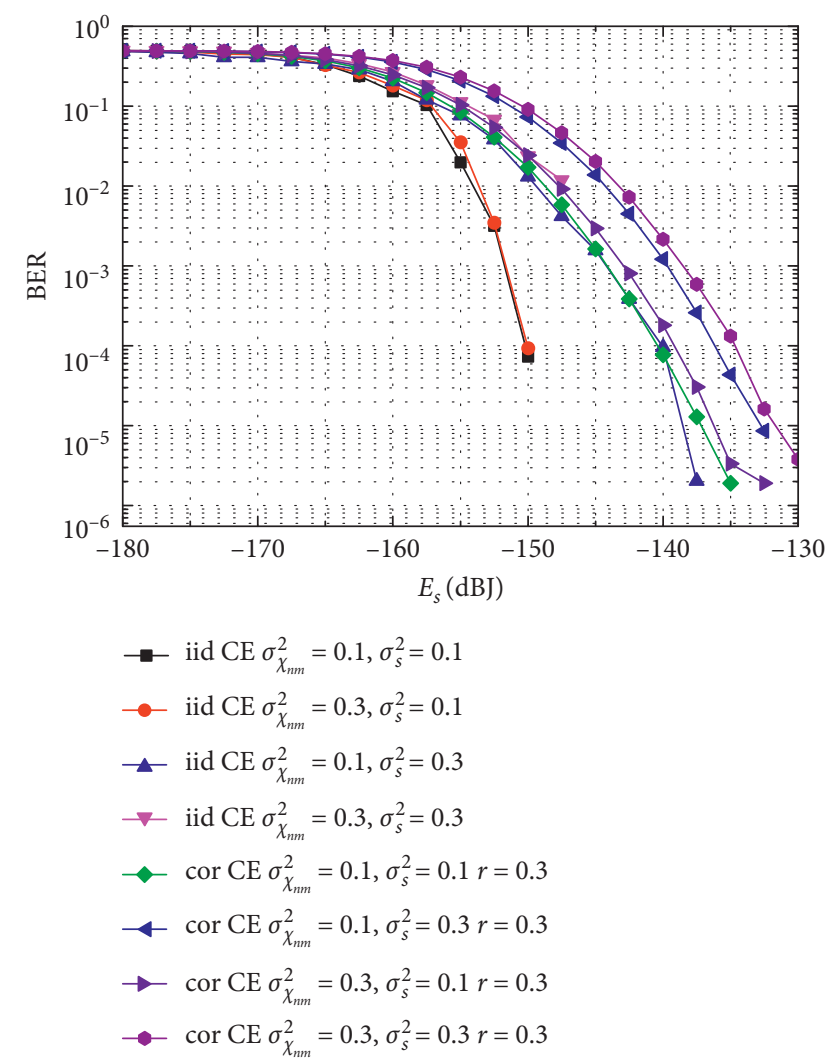

FIGURE 5: BER with combined effects over correlated channel for different atmospheric turbulence and pointing error jitter variance.

Figure 9 shows the approximate ergodic channel capacity of $2 \times 2$ and $4 \times 4$ WOC MIMO system over independent channel. It is evident from the plot that the CE will seriously impact on the ergodic channel capacity. For example, when $E_{s}=-190 \mathrm{dBJ}$, the ergodic channel capacity

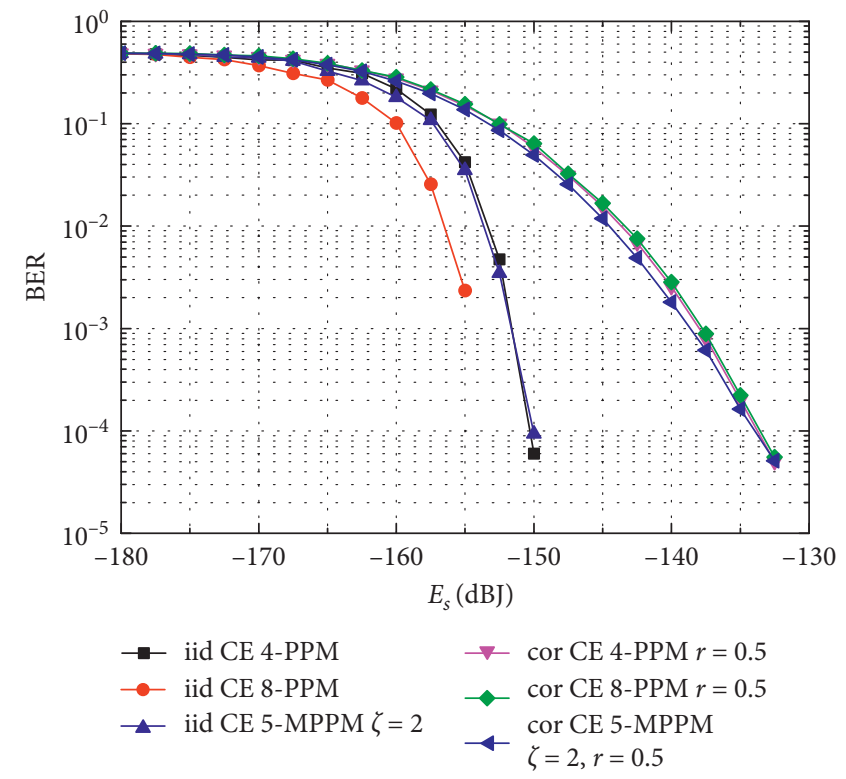

FIGURE 6: BER performance of different modulation methods in a2 $\times 2$ MIMO system.



FIgURE 7: Ergodic channel capacity of different modulation methods in a $2 \times 2$ MIMO system.

reduced by $2.64 \mathrm{bits} / \mathrm{channel}$ compared with the Per case in the $4 \times 4$ system. It should be noted that the influence of pointing error is more significant than atmospheric attenuation and atmospheric turbulence. The reduction caused by pointing error is about $2.27 \mathrm{bits} / \mathrm{channel}$.

Figure 10 shows the approximate ergodic channel capacity of a $4 \times 4$ WOC MIMO system under combined effects over correlated channel with different correlation coefficients. It can be observed that the ergodic channel capacity decreases with the increasing of correlation coefficient. For example, when the correlation coefficients are $0,0.3,0.5$, and 0.8 , the thresholds of capacity saturation are $-167.5 \mathrm{dBJ},-162.5 \mathrm{dBJ},-155 \mathrm{dBJ}$, and 




FIGURE 8: Theoretical and approximate ergodic channel capacity with different number of "on" slots.

$-135 \mathrm{dBJ}$, respectively. Furthermore, when the ergodic channel capacity reaches its saturation, the power loss caused by the combined effects is $20 \mathrm{dBJ}$, while the power loss caused by the strong correlation is $22.5 \mathrm{dBJ}$, which is greater than that of combined effects. Therefore, it can be considered that strong correlation has more effect on ergodic channel capacity than combined effects.

Figure 11 shows the approximate ergodic channel capacity with different atmospheric turbulence and pointing error jitter variance over correlated channel. The ergodic channel capacity decreases with the increasing of $\sigma_{\chi_{n m}}^{2}$ and $\sigma_{s}^{2}$, where $\sigma_{s}^{2}$ plays a more important role in the process. For example, at $E_{s}=-172.5 \mathrm{dBJ}$, the ergodic channel capacity decreases by about $0.034 \mathrm{bits} / \mathrm{channel}$ and $0.467 \mathrm{bits} / \mathrm{chan}-$ nel, when $\sigma_{x}^{2}$ and $\sigma_{s}^{2}$ increase from 0.1 to 0.3 , respectively. As a result, the increase of $\sigma_{s}^{2}$ has a greater impact on the ergodic channel capacity of the WOC MIMO system than the increase of $\sigma_{\chi_{m m}}^{2}$.

\section{Conclusions}

The MPPM-coded WOC MIMO system performance over correlated fading channel under combined effects of atmospheric attenuation, atmospheric turbulence, and pointing error is discussed. The approximate expressions of BER and ergodic channel capacity are proposed to reduce the computational complexity. The research results indicate that (1) the pointing error is the prominent influence factor in independent and weak correlated channel and (2) the performance degradation caused by high channel correlation coefficient is more than that of pointing error in strong correlated channel. Therefore, in practical applications, the number and spacing of lasers should be reasonably designed

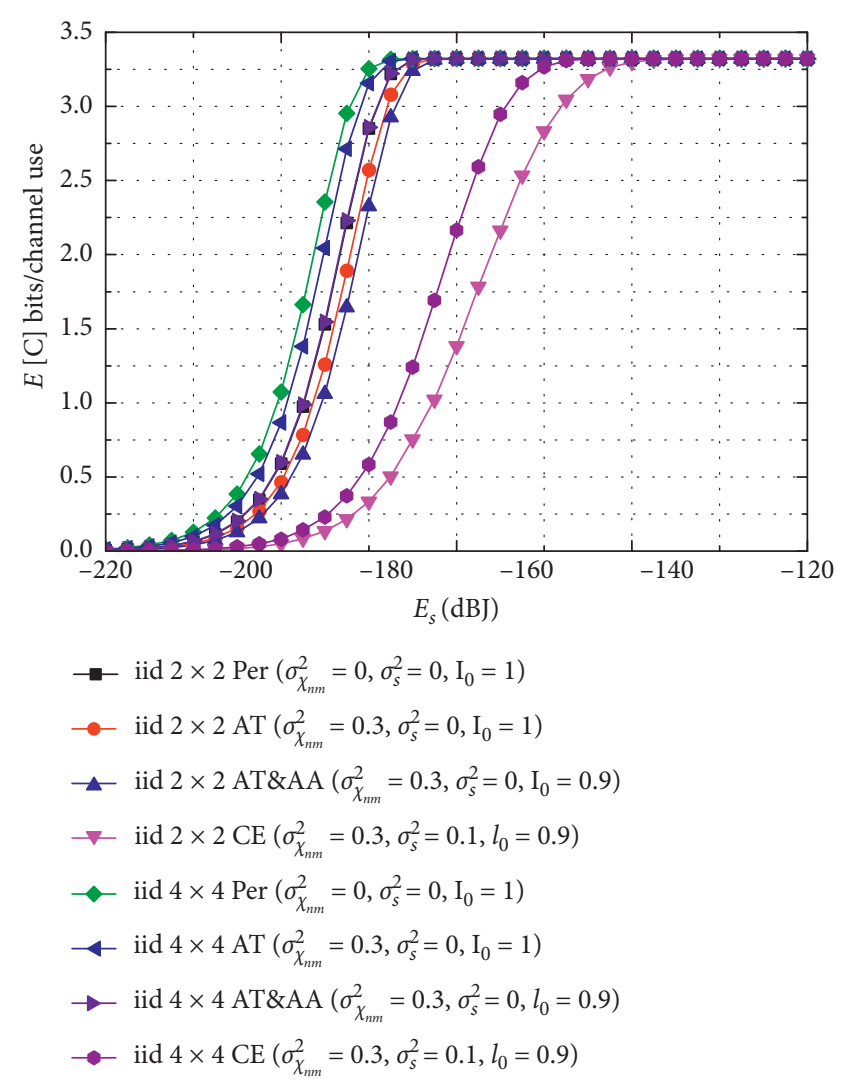

FIgURE 9: Ergodic channel capacity with different conditions over independent channel.

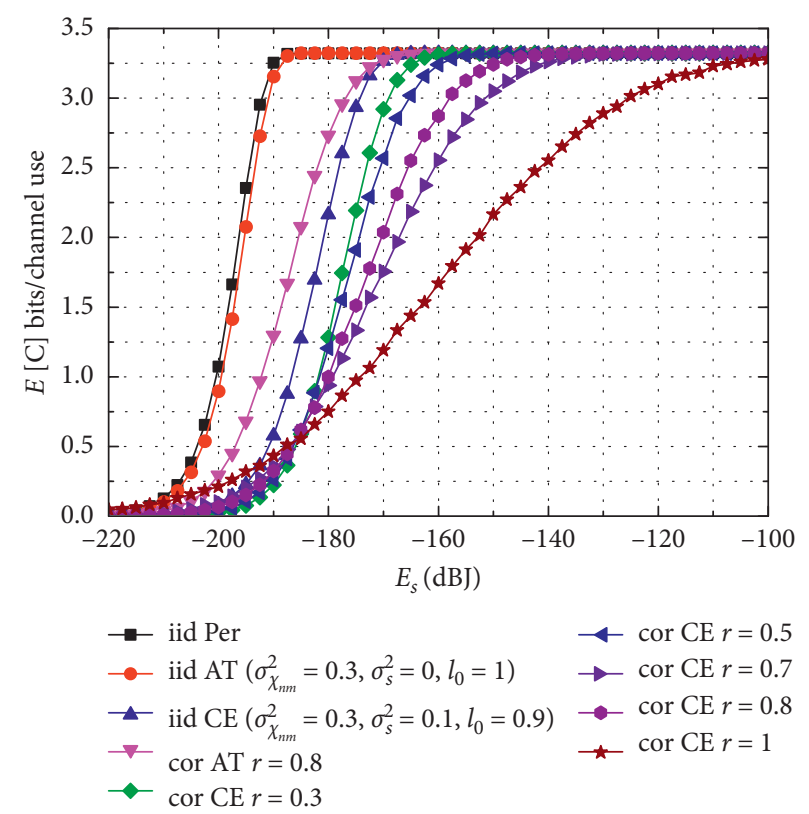

Figure 10: Ergodic channel capacity under combined effects over correlated channel for different correlation coefficients.

to minimize the spatial correlation of subchannels. Additionally, the system performance can be further improved by 


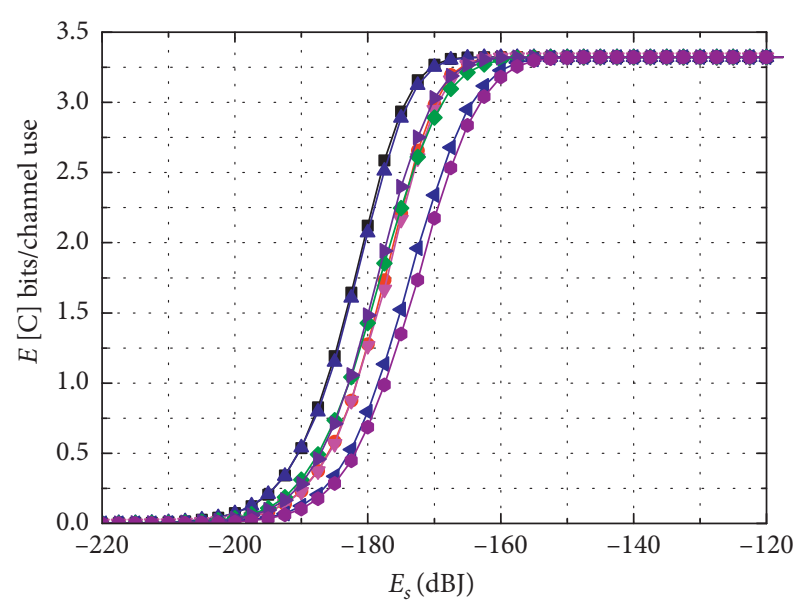

$$
\begin{aligned}
& \rightarrow-\operatorname{iid~CE~} \sigma_{\chi_{n m}}^{2}=0.1, \sigma_{s}^{2}=0.1 \\
& \longrightarrow \operatorname{iid~CE~} \sigma_{\chi_{n m}}^{2}=0.1, \sigma_{s}^{2}=0.3 \\
& \neg \operatorname{iid~CE~} \sigma_{\chi_{n m}}^{2}=0.3, \sigma_{s}^{2}=0.1 \\
& \neg-\operatorname{iid~CE~} \sigma_{\chi_{n m}}^{2}=0.3, \sigma_{s}^{2}=0.3 \\
& \neg-\operatorname{cor} \mathrm{CE} \sigma_{\chi_{n m}}^{2}=0.3, \sigma_{s}^{2}=0.1 r=0.3 \\
& \multimap \operatorname{cor} \mathrm{CE} \sigma_{\chi_{n m}}^{2}=0.1, \sigma_{s}^{2}=0.3 r=0.3 \\
& \rightarrow \operatorname{cor} \mathrm{CE} \sigma_{\chi_{n m}}^{2}=0.1, \sigma_{s}^{2}=0.1 r=0.3 \\
& \rightarrow \operatorname{cor} \mathrm{CE} \sigma_{\chi_{n m}}^{2}=0.3, \sigma_{s}^{2}=0.3 r=0.3
\end{aligned}
$$

FIGURE 11: Ergodic channel capacity with combined effects over correlated channel for different atmospheric turbulence and pointing error jitter variance.

using the beam tracking systems to restrain the pointing error.

\section{Data Availability}

The data used to support the findings of this study are available from the corresponding author upon request.

\section{Conflicts of Interest}

The authors declare that there are no conflicts of interest regarding the publication of this paper.

\section{Acknowledgments}

This work was funded by the National Natural Science Foundation of China (NSFC) (61861026, 61875080, and 61465007) and Ph.D Foundation of Lanzhou University of Technology (03-061616).

\section{References}

[1] P. W. Berenguer, P. Hellwig, D. Schulz et al., "Real-time optical wireless mobile communication with high physical layer reliability," Journal of Lightwave Technology, vol. 37, no. 6, pp. 1638-1646, 2019.

[2] N. Joshi and P. K. Sharma, "Performance of wireless optical communication in S-distributed turbulence," IEEE Photonics Technology Letters, vol. 28, no. 2, pp. 151-154, 2016.
[3] E. Balti and B. K. Johnson, "Asymmetric RF/FSO relaying with HPA non-linearities and feedback delay constraints," IEEE Transactions on Wireless Communications, pp. 1-36, 2019.

[4] G. Aarthi, K. Prabu, and G. R. Reddy, “Aperture averaging effects on the average spectral efficiency of FSO links over turbulence channel with pointing errors," Optics Communications, vol. 385, pp. 136-142, 2017.

[5] M. T. Dabiri, M. J. Saber, and S. M. S. Sadough, "On the performance of multiplexing FSO MIMO links in log-normal fading with pointing errors," Journal of Optical Communications and Networking, vol. 9, no. 11, pp. 974-983, 2017.

[6] L. Han and Y. You, "Performance of multiple input multiple output free space optical communication under atmospheric turbulence and atmospheric attenuation," Chinese Journal of Lasers, vol. 43, no. 7, Article ID 0706004, 2016.

[7] I. A. Alimi, A. M. Abdalla, J. Rodriguez, P. P. Monteiro, and A. L. Teixeira, "Spatial interpolated lookup tables (LUTs) models for ergodic capacity of MIMO FSO systems," IEEE Photonics Technology Letters, vol. 29, no. 7, pp. 583-586, 2017.

[8] P. K. Sharma, A. Bansal, and P. Garg, "Relay assisted Bi-directional communication in generalized turbulence fading," Journal of Lightwave Technology, vol. 33, no. 1, pp. 133-139, 2015.

[9] A. Garacia-Zambrana, B. Castillo-Vazquez, and C. CastilloVazquez, "Asymptotic error-rate analysis of FSO links using transmit laser selection over gamma-gamma atmospheric turbulence channels with pointing errors," Optics Express, vol. 20, no. 3, pp. 2096-2109, 2012.

[10] M. R. Bhatnagar and Z. Ghssemlooy, “"Performance analysis of Gamma-Gamma fading FSO MIMO links with pointing errors," Journal of Lightwave Technology, vol. 34, no. 9, pp. 2158-2169, 2016.

[11] T. Ozbilgin and M. Koca, "Inter-aperture correlation in MIMO free space optical systems," Optics Communications, vol. 353, pp. 139-146, 2015.

[12] M. V. Jamali, J. A. Salehi, and F. Akhoundi, "Performance studies of underwater WOC communication systems with spatial diversity: MIMO scheme," IEEE Transactions on Communications, vol. 65, no. 3, pp. 1176-1192, 2017.

[13] J. A. Anguita, M. A. Neifeld, and B. V. Vasic, "Spatial correlation and irradiance statistics in a multiple-beam terrestrial free-space optical communication link," Applied Optics, vol. 46, no. 26, pp. 6561-6571, 2007.

[14] Z. Chen, S. Yu, T. Wang, G. Wu, S. Wang, and W. Gu, "Channel correlation in aperture receiver diversity systems for free-space optical communication," Journal of Optics, vol. 14, no. 12, Article ID 125710, 2012.

[15] G. Yang, S. Bourennane, Z. Ghassemlooy, and M. A. Khalighi, "Performance analysis of space-diversity free-space optical systems over the correlated Gamma-Gamma fading channel using Padé approximation method," IET Communications, vol. 8, no. 13, pp. 2246-2255, 2014.

[16] M. A. Khalighi, N. Schwartz, N. Aitamer, and S. Bourennane, "Fading reduction by aperture averaging and spatial diversity in optical wireless systems," IEEE/OSA Journal of Optical Communications Networking, vol. 1, no. 6, pp. 580-593, 2009.

[17] N. Wu, X. Wang, and H. Dai, "Performance of indoor visible light systems using OOK and PPM modulations under multipath channels," in Proceedings of the 2013 2nd International Workshop on Optical Wireless Communications (IWOW), pp. 84-88, Newcastle Upon Tyne, UK, October 2013. 
[18] X. Ke and Z. Yin, Coding Theory in Wireless Laser Communication System, Science Press, Beijing, China, 2008.

[19] P. Wang, J. Qin, L. Guo, and Y. Yang, "BER performance of FSO limited by shot and thermal noise over exponentiated weibull fading channels," IEEE Photonics Technology Letters, vol. 28, no. 3, pp. 252-255, 2016.

[20] H. Sugiyama and K. Nosu, "MPPM: a method for improving the band-utilization efficiency in optical PPM," Journal of Lightwave Technology, vol. 7, no. 3, pp. 465-472, 1989.

[21] R. Zhou, T. Cao, Y. Yang et al., "Symbol error rate performance analysis of soft-decision, decoded MPPM free space optical system over exponentiated Weibull fading channels," Chinese Optics Letters, vol. 15, no. 5, Article ID 050602, 2017.

[22] M. Hassan, S. Shapsough, T. Landolsi, and A. F. Elrefaie, "Error performance study of MPPM optical communication systems with finite extinction ratios," in Proceedings of the 2016 International Conference on Industrial Informatics \& Computer Systems (CIICS), Sharjah, UAE, March 2016.

[23] J. M. Garrido-Balsells, F. J. López-González, and A. Juradonavas, "General closed-form bit-error rate expressions for coded $M$-distributed atmospheric optical communications," Optics Letters, vol. 40, no. 13, pp. 2937-2940, 2015.

[24] I. I. Kim, B. Mcarthur, and E. J. Korevaar, "Comparison of laser beam propagation at $785 \mathrm{~nm}$ and $1550 \mathrm{~nm}$ in fog and haze for optical wireless communications," Proceedings of SPIE, vol. 4214, no. 2, pp. 26-37, 2001.

[25] A. S. El-Wakeel, N. A. Mohammed, and M. H. Aly, "Free space optical communications system performance under atmospheric scattering and turbulence for 850 and nm operation," Applied Optics, vol. 55, no. 26, pp. 7276-7286, 2016.

[26] C. Martin and B. Ottersten, "Asymptotic eigenvalue distributions and capacity for MIMO channels under correlated fading," IEEE Transactions on Wireless Communications, vol. 3, no. 4, pp. 1350-1359, 2004.

[27] A. A. Abu-Dayya and N. C. Beaulieu, "Outage probabilities in the presence of correlated lognormal interferers," IEEE Transations Vehicular Technology, vol. 43, no. 1, pp. 164-173, 1994. 